\title{
A POLYNOMIAL ROOTING APPROACH FOR SYNCHRONIZATION IN MULTIPATH CHANNELS USING ANTENNA ARRAYS
}

\author{
Gonzalo Seco, A. Lee Swindlehurst! Juan A. Fernández-Rubio* \\ *Dept. of Signal Theory \& Communications, Univ. Politècnica de Catalunya, Barcelona, SPAIN. \\ † Dept. of Electrical \& Computer Engineering, Brigham Young University, Provo, Utah, USA. \\ email: gonzalo/juan@gps.tsc.upc.es, swindle@ee.byu.edu
}

\begin{abstract}
The estimation of the delay of a known training signal received by an antenna array in a multipath channel is addressed. The effect of the co-channel interference is taken into account by including a term with unknown spatial correlation. The channel is modeled as an unstructured FIR filter. The exact maximum likelihood (ML) solution for this problem is derived, but it does not have a simple dependence on the delay. An approximate estimator that is asymptotically equivalent to the exact one is presented. Using an appropriate reparameterization, it is shown that the delay estimate is obtained by rooting a low-order polynomial, which may be of interest in applications where fast feedforward synchronization is needed.
\end{abstract}

\section{INTRODUCTION}

Time-delay estimation or timing synchronization is a key task in diverse areas, such as radar, sonar and communications. Accurate chip/symbol synchronization is especially important in systems employing time-division multiple access (TDMA) or asynchronous burst transmissions. Also, most multiuser detectors for code-division multiple access (CDMA) require reliable estimates of the users' code timings in order to operate acceptably in near-far environments [1]. In addition, Global Navigation Satellite Systems (GNNS) arouse great interest at present. In these systems, accurate time-delay estimation is fundamental, since it is the key to obtain sub-meter accuracies in location estimates.

There is a vast literature on single antenna synchronization methods for both additive white Gaussian noise (AWGN) channels and multipath channels [2]. However, the performance of these methods is limited when strong co-channel interference (CCI) is present. For this reason, an important effort is being conducted to derive time-delay estimators that make efficient use of antenna arrays in interference limited scenarios. Following an approach that has already been applied successfully to this and other problem, all the components contributing to the noise and CCI

* Partial support provided by the Catalan and Spanish Governments under grants 1997FI 00755 APDT, TIC98-0412, TIC98-0703, TIC99-0849, and CIRIT 1998SGR-00081.

† Partial support provided by U.S. National Science Foundation Wireless Initiative Grant CCR 99-79452, and by the U.S. Office of Naval Research under Grant N00014-99-1-0692. (i.e., multi-access interference (MAI), external interference, etc.) are modeled as a Gaussian term with unknown and arbitrary spatial correlation matrix $[3,4,5]$. This model allows us to develop a metric that takes the spatial characteristics of the CCI into account, and we believe that this offers an excellent trade-off between model realism and computational complexity. A more detailed description of the CCI, e.g. using the finite alphabet property of the MAI, may result in an improved performance, but at the expense of avoiding a simple expression for the estimator. On the other hand, several computationally attractive algorithms have been derived under the usual assumption that the CCI is spatially white [6]. However, the resulting algorithms are not suited for situations involving strong CCI. The estimation of the spatial covariance of the CCI is only possible if a training sequence is received. Therefore, the estimator presented herein and those that assume the same model for the CCI can only operate in data-aided or decisiondirected mode. The assumption that the signal shape is known should not be a too stringent one, since most communications or satellite navigation systems transmit certain training sequences and, subsequently the estimator can be switched to a decision-directed mode. In addition, in radar and sonar systems the shape of the received signal coincides with that of the transmitted one.

Some methods have focused on determining the timedelays of the multipath components together with some other parameters, for instance the directions of arrival (DOA), describing the channel $[7,8,9]$. Those methods exploit the full space-time structure of the multipath. Except for some cases that resort to a particular configuration of the antenna array, the primary drawback of these approaches is that complicated search procedures are required to estimate the desired parameters. Moreover, when DOA estimates are to be estimated, it is necessary to have a calibrated antenna array, which is a restrictive assumption. For these reasons, we will use an unstructured model for the channel. Although this leads to an increase in the number of unknowns with respect to a parameterized model, the dependence on the channel is linear and it can be estimated in closed form, as in [3].

A number of techniques that estimate the delays of each of the received replicas and assume that the value of each delay is arbitrary have been developed. However, receivers usually combine the different rays of the received signal using a RAKE structure. This structure can be viewed as a 
bank of filters, with a fixed delay (typically the inverse of the bandwidth or a fraction thereof) between each pair of filters. Hence, it seems logical to extend this structure also to the timing synchronization. The method proposed in this paper is based on the estimator in $[4,5]$. The difference lies in that now a fixed separation between the received replicas is assumed, thereby only the absolute delay of the whole set of replicas has to be estimated. The interesting consequence of this model is that the delay can be obtained by finding the roots of a low-order polynomial, for which computationally efficient algorithms exist. Consequently, the method is specially tailored to applications where fast (feedforward) synchronization of the received signal is needed [2].

\section{DATA MODEL}

The signal received by an arbitrary $m$ element array is modeled as

$$
\mathbf{y}[n]=\sum_{k=1}^{d} \mathbf{h}_{k} s\left(n T_{s}-\tau-(k-1) T_{0}\right)+\mathbf{e}[n]
$$

where $T_{s}$ is the sampling period, $\left\{\mathbf{h}_{k}\right\}$ are the FIR channel coefficient vectors and $d$ is the temporal length of the channel. $T_{0}$ is the temporal spacing of the FIR channel and can be freely chosen, together with $d$, when setting up the model (1). The transmitted signal $s(t)$ is assumed to be known to within the scalar time delay parameter $\tau$. If $N$ samples are collected, they all may be grouped together as follows:

$$
\mathbf{Y}=\left[\begin{array}{llll}
\mathbf{y}[1] & \mathbf{y}[2] & \ldots & \mathbf{y}[N]
\end{array}\right]=\mathbf{H} \mathbf{S}(\tau)+\mathbf{E}
$$

where $\mathbf{E}$ is formed identically to $\mathbf{Y}$ and

$$
\mathbf{H}=\left[\begin{array}{llll}
\mathbf{h}_{1} & \mathbf{h}_{2} & \ldots & \mathbf{h}_{d}
\end{array}\right]
$$

The $m \times d$ matrix $\mathbf{H}$ represents the single-input-multipleoutput (SIMO) channel for the signal of interest. The $p, q-$ th element of the matrix $\mathbf{S}(\tau)$ is $s\left(q T_{s}-\tau-(p-1) T_{0}\right)$. The term e $[n]$, which gathers the noise and all other $\mathrm{CCI}$, is modeled as a complex, circularly-symmetric, zero-mean Gaussian process. It is assumed to be temporally white and spatially colored with an arbitrary unknown correlation matrix:

$$
\mathcal{E}\left\{\mathbf{e}[n] \mathbf{e}^{*}[m]\right\}=\mathbf{Q} \delta_{n, m}
$$

where $(\cdot)^{*}$ denotes the complex conjugate transpose operation. While such a model for $e[n]$ is clearly only approximate, it captures the most significant effects of the noise and interference, and leads to tractable algorithms. For the asymptotic results in the next section to be valid, the following additional assumption is needed: $s(t)$ is a bandlimited finite-average-power signal, and the sampling period $T_{s}$ satisfies the Nyquist criterion.

Note that though the pulse shaping filter could be factored into the channel matrix, as in [8], we assume herein that the elements of $\mathbf{S}(\tau)$ are samples of the continuous modulated waveform $s(t)$. As such, the matrix $H$ only describes the propagation effects of the channel, and $\tau$ is a continuous-valued parameter. This modeling premise is different to those usually taken in other work addressing the equalization of FIR channels rather than the synchronization.

The model in (1) is closely related to that employed in other methods that attempt to estimate the delays of the different arrivals, such as $[7,6,4,5]$. In those cases, the received vector model consists of the contribution of $L$ arrivals as follows

$$
\mathbf{Y}=\mathbf{A} \tilde{\mathbf{S}}(\boldsymbol{\tau})+\mathbf{E}
$$

where

$$
\begin{aligned}
\boldsymbol{\tau} & =\left[\begin{array}{llll}
\tau_{1} & \ldots & \tau_{L}
\end{array}\right]^{T} \quad \mathbf{A}=\left[\begin{array}{lll}
\mathbf{a}_{1} & \ldots & \mathbf{a}_{L}
\end{array}\right] \\
\mathbf{s}\left(\tau_{l}\right) & =\left[\begin{array}{llll}
s\left(T_{s}-\tau_{l}\right) & \ldots & s\left(N T_{s}-\tau_{l}\right)
\end{array}\right] \\
\tilde{\mathbf{S}}(\boldsymbol{\tau}) & =\left[\begin{array}{llll}
\mathbf{s}^{T}\left(\tau_{1}\right) & \ldots & \mathbf{s}^{T}\left(\tau_{L}\right)
\end{array}\right]^{T} .
\end{aligned}
$$

The columns of the matrix $\mathbf{A}$ are the spatial signatures of the different arrivals. Assuming that the signal $s(t)$ is bandlimited and $T_{0}$ satisfies the Nyquist criterion, each row of the matrix $\tilde{\mathbf{S}}(\boldsymbol{\tau})$ can be expressed as a linear combination of the elements of $\mathbf{S}(\tau)$ [10]. Therefore, there exist a $L \times d$ interpolating matrix $\mathbf{T}$ that satisfies

$$
\tilde{\mathbf{S}}(\boldsymbol{\tau})=\mathbf{T S}(\tau)
$$

For the equality (9) to be exact in a general case, the column dimension of $\mathbf{T}$, that is $d$, should be infinite. However, very good approximations can be obtained for finite $d[10]$. Finally, identifying the channel matrix as $\mathbf{H}=\mathbf{A} \mathbf{T}$, the relationship between the models in (1) and (5) becomes apparent.

\section{MAXIMUM LIKELIHOOD ESTIMATOR AND ASYMPTOTICALLY EQUIVALENT APPROXIMATION}

Under the model described above, the negative log-likelihood function of the data $\mathbf{Y}$ in (2) is given by (to within irrelevant constants) ${ }^{1}$

$$
\Lambda(\tau, \mathbf{H}, \mathbf{Q})=\log |\mathbf{Q}|+\operatorname{Tr}\left\{\mathbf{C}(\tau, \mathbf{H}) \mathbf{Q}^{-1}\right\},
$$

where

$$
\begin{aligned}
& \mathbf{C}(\tau, \mathbf{H})=\hat{\mathbf{R}}_{y y}-\mathbf{H} \hat{\mathbf{R}}_{y s}^{*}(\tau)-\hat{\mathbf{R}}_{y s}(\tau) \mathbf{H}^{*}+\mathbf{H} \hat{\mathbf{R}}_{s s}(\tau) \mathbf{H}^{*} \\
& \hat{\mathbf{R}}_{y y}=\frac{1}{N} \mathbf{Y} \mathbf{Y}^{*} \quad \hat{\mathbf{R}}_{y s}(\tau)=\frac{1}{N} \mathbf{Y} \mathbf{S}^{*}(\tau) \\
& \hat{\mathbf{R}}_{s s}(\tau)=\frac{1}{N} \mathbf{S}(\tau) \mathbf{S}^{*}(\tau)
\end{aligned}
$$

Since $\mathbf{H}$ and $\mathbf{Q}$ are taken as unstructured deterministic matrices, the minimization of (10) may be performed explicitly with respect to them. Their ML estimates may be expressed as

$$
\begin{aligned}
\hat{\mathbf{H}}_{M L}(\tau) & =\hat{\mathbf{R}}_{y s}(\tau) \hat{\mathbf{R}}_{s s}^{-1}(\tau) \\
\hat{\mathbf{Q}}_{M L}(\tau) & =\hat{\mathbf{R}}_{y y}-\hat{\mathbf{R}}_{y s}(\tau) \hat{\mathbf{R}}_{s s}^{-1}(\tau) \hat{\mathbf{R}}_{y s}^{*}(\tau) .
\end{aligned}
$$

$1|\cdot|$ and $\operatorname{Tr}\{\cdot\}$ denote the determinant and the trace of a matrix, respectively. 
Ignoring parameter independent constants, the resulting ML criterion for $\tau$ is the minimizing argument of

$$
f(\tau)=\log |\mathbf{I}-\mathbf{B}(\tau)|
$$

where

$$
\begin{aligned}
\mathbf{B}(\tau) & =\frac{1}{N} \hat{\mathbf{R}}_{y y}^{-\frac{1}{2}} \mathbf{Y} \mathbf{P}_{\mathbf{S}^{*}(\tau)} \mathbf{Y}^{*} \hat{\mathbf{R}}_{y y}^{-\frac{1}{2}} \\
\mathbf{P}_{\mathbf{S}^{*}(\tau)} & =\mathbf{S}^{*}(\tau)\left(\mathbf{S}(\tau) \mathbf{S}^{*}(\tau)\right)^{-1} \mathbf{S}(\tau) .
\end{aligned}
$$

If the noise had been assumed spatially white (i.e. $\mathbf{Q}=$ $\left.\sigma^{2} I\right)$, the ML cost function, in place of (15), would have been

$$
f^{w}(\tau)=-\frac{1}{N} \operatorname{Tr}\left\{\mathbf{Y} \mathbf{P}_{\mathbf{S}^{*}(\tau)} \mathbf{Y}^{*}\right\} .
$$

Since the dependence of this cost function on the projection matrix $\mathbf{P}_{\mathbf{S}}$ is linear, the algorithm presented in the next section could be applied in order to find the minimum of (18) by rooting a polynomial. This interesting algorithm is not directly applicable to (15) because of the determinant operator. However, based on the results of $[4,5]$, it is straightforward to build a cost function that is linear in the projector $\mathbf{P}_{\mathbf{S}}$ * and yields asymptotically (large $N$, throughout the paper) equivalent estimates to those provided by $f(\tau)$. This alternative cost function to be minimized is

$$
g(\tau, \hat{\mathbf{W}})=-\operatorname{Tr}\{\hat{\mathbf{W}} \mathbf{B}(\tau)\}
$$

The weighting matrix $\hat{\mathbf{W}}$ is computed as

$$
\hat{\mathbf{W}}=(\mathbf{I}-\mathbf{B}(\hat{\tau}))^{-1}
$$

where $\hat{\tau}$ is a consistent estimate of the true delay. This initial consistent estimate, that is when a previous estimate is not available to compute the weighting matrix, is simply obtained as the minimizing argument of $g(\tau, I)$. Following the development in $[4,5]$, it can be shown that both (15) and (19) provide, under mild conditions and in absence of modeling errors, consistent and asymptotically efficient estimates. Note that it can be argued that since $N$ is the length of the training sequence, we will never reach asymptotics in $N$. However, the discussion above is completely meaningful because the numerical results show that the asymptotic behaviour is reached for rather modest sample sizes. It is not difficult to show that the CRB for the problem at hand is

$\mathrm{CRB}^{-1}(\tau)=2 \operatorname{Tr}\left\{\left(\tilde{\mathbf{D}}(\boldsymbol{\tau}) \mathbf{P}_{\tilde{\mathbf{S}}^{*}(\tau)}^{1} \tilde{\mathbf{D}}^{*}(\tau)\right)\left(\mathbf{H}^{*} \mathbf{Q}^{-1} \mathbf{H}\right)\right\}$,

where the matrices $\tilde{\mathbf{S}}$ and $\tilde{\mathbf{D}}$ (which is the derivative of the former) are evaluated at

$$
\tau=\left[\begin{array}{llll}
\tau & \tau+T_{0} & \ldots & \tau+(d-1) T_{0}
\end{array}\right]^{T} .
$$

\section{POLYNOMIAL ROOTING APPROACH}

At this point we are concerned with the minimization of the following general expression

$g(\tau, \mathbf{W})=-\frac{1}{N} \operatorname{Tr}\left\{\mathbf{W}^{1 / 2} \hat{\mathbf{R}}_{y y}^{-1 / 2} \mathbf{Y} \mathbf{P}_{\mathbf{S}^{*}(\tau)} \mathbf{Y}^{*} \hat{\mathbf{R}}_{y y}^{-1 / 2} \mathbf{W}^{1 / 2}\right\}$
For appropriate choices of $\mathbf{W}$, this expression represents the asymptotically efficient and the consistent estimators for correlated noise $(g(\tau, \hat{\mathbf{W}})$ and $g(\tau, \mathbf{I}))$, and the whitenoise ML estimator $\left(f^{w}(\tau)\right)$. Now, the $N$ temporal samples are transformed into the frequency domain using the DFT, so that the signal approximately satisfies the following relationship ${ }^{2}$

$$
\mathbf{S}^{*}(\tau)=\mathbf{S}_{\omega}^{*} \mathbf{V}(\tau)
$$

where $\mathbf{S}_{\omega}$ is a diagonal matrix whose entries are the DFT of the samples $\left[s\left(T_{s}\right), \cdots, s\left(N T_{s}\right)\right]$, and

$$
\begin{aligned}
\mathbf{V}(\tau) & =\left[\begin{array}{llll}
\mathbf{v}(\tau) & \mathbf{v}\left(\tau+T_{0}\right) & \cdots & \mathbf{v}\left(\tau+(d-1) T_{0}\right)
\end{array}\right] \\
\mathbf{v}(\tau) & =\left[\begin{array}{lll}
\exp \left(j \omega_{1} \tau\right) & \cdots & \exp \left(j \omega_{N} \tau\right)
\end{array}\right]^{T} \\
\omega_{i} & =\frac{2 \pi}{N T_{s}}\left(i-1-\text { floor }\left(\frac{N}{2}\right)\right)
\end{aligned}
$$

The criterion in (21) may be expressed as a function of $x \triangleq \exp \left(j 2 \pi \tau / N T_{s}\right)$, resulting in a polynomial in $x$ of order $2 N-2$, since $\left(\mathbf{V}^{*}(\tau) \mathbf{S}_{\omega} \mathbf{S}_{\omega}^{*} \mathbf{V}(\tau)\right.$ does not depend on $x$. This approach lacks of interest because $N$ is generally large. Below we describe a method that leads to the rooting of polynomials of order $2 d$, and it is natural that $d \ll N$.

Let the elements of the vector $\mathbf{p}=\left[p_{0} \cdots p_{d}\right]^{T}$ be taken from the coefficients of the polynomial

$$
p(z)=p_{0} z^{d}+p_{1} z^{d-1}+\cdots+p_{d}
$$

whose roots are:

$$
\left\{r^{0} x, r^{1} x, \cdots, r^{(d-1)} x\right\},
$$

where $r \triangleq \exp \left(j 2 \pi T_{0} / N T_{s}\right)$. If we define

$$
\begin{aligned}
& \mathbf{F}=\mathbf{S}_{\omega}^{-*} \mathbf{Y}^{*} \hat{\mathbf{R}}_{y y}^{-\frac{1}{2}} \mathbf{W}^{\frac{1}{2}} / \sqrt{N} \\
& \mathbf{\Psi}=\left(\mathbf{P}^{*} \mathbf{S}_{\omega}^{-*} \mathbf{S}_{\omega}^{-1} \mathbf{P}\right)^{-1}
\end{aligned}
$$

and build the $N \times N-d$ matrix

$$
\mathbf{P}=\left[\begin{array}{ccccccc}
p_{d d} & p_{d-1} & \cdots & p_{0} & 0 & & \\
0 & p_{d} & p_{d-1} & \cdots & p_{0} & 0 & \\
& \ddots & \ddots & \ddots & \ddots & \ddots & 0 \\
& & 0 & p_{d} & p_{d-1} & \cdots & p_{0}
\end{array}\right]^{*}
$$

then minimizing (21) is equivalent to minimizing [6]

$$
\tilde{g}(\mathbf{p}, \mathbf{W})=\operatorname{Tr}\left\{\mathbf{F}^{*} \mathbf{P} \mathbf{\Psi} \mathbf{P}^{*} \mathbf{F}\right\} .
$$

It can be readily shown that the vector $p$ satisfies

$$
\mathbf{p}=\mathbf{K} \mathbf{t}(x)
$$

where $\mathbf{K}$ is diagonal matrix whose elements are the coeffcients of the polynomial $p(z)$ for the case $x=1$, and

$$
\mathbf{t}(x)=\left[\begin{array}{llll}
1 & x & \cdots & x^{d}
\end{array}\right]^{T} .
$$

\footnotetext{
${ }^{2}$ The same notation is used for both the time and frequency domains because the DFT is an unitary transformation and therefore, the estimators presented in section 3 are identically applicable in the frequency domain.
} 
Therefore, if the term $\Psi$ is held fixed, the cost function in (30) can be written as a polynomial in $x$ of order $2 d$, as follows:

$$
\tilde{g}(x, \mathbf{W})=\mathbf{t}^{T}(1 / x) \mathbf{K}^{*} \mathbf{C K} \mathbf{t}(x)
$$

for some matrix $\mathbf{C}$ obtained from $\mathbf{F}$ and $\Psi$. For sake of the brevity the explicit form of $\mathbf{C}$ is omitted. The minimum of $\tilde{g}(x, \mathbf{W})$ on the unit circle is computed by first finding the roots of its derivative. Next, (33) is evaluated at the set of roots that lie on the unit circle, and the one giving the minimum is selected. Using this root and the definition of $x$, the delay estimate is easily obtained. This procedure is repeated until convergence or failure conditions are satisfied (e.g., in the simulations these conditions are: change in $x$ smaller than $10^{-4}$, number iterations larger than 50 ). At each iteration, the matrix $\boldsymbol{\Psi}$ is recomputed using the previous estimate of $x$; and in the first iteration, $\Psi$ is taken equal to the identity.

An essential feature of this algorithm is that the inverse matrix operation needed in the computation of $\Psi$ needs to be calculated only once, and it can be done off-line since the matrix to be inverted depends exclusively on some design parameters. This follows from the fact that $\Psi$ can be decomposed as

$$
\Psi=\mathbf{U} \Psi_{1} \mathbf{U}^{-1}
$$

where $\Psi_{1}$ is the value of $\Psi$ for $x=1$, and

$$
\mathrm{U}=\operatorname{diag}\left\{1, x, \ldots, x^{N-d+1}\right\} \text {. }
$$

Therefore, the update of $\Psi$ at every iteration only involves the left and right-hand product of a fixed matrix by a diagonal one that solely depends on $x$.

\section{NUMERICAL RESULTS}

We analyze the performance of the estimators proposed in this paper, and compare it with the Cramér-Rao Bound (CRB). Specifically, we consider the exact ML estimator for the colored-noise case given by (15), its approximation in (19) and the ML estimator for the white-noise case in (18). The cost function of the first one is minimized by means of a search. Whereas, the polynomial rooting algorithm in section 4 is applied to the latter two. In the case of the approximate ML estimator, we have chosen to update the matrix $\hat{\mathbf{W}}$, together with $\Psi$, at each iteration of the algorithm. The RMSE (root mean square error) are computed from 500 Monte Carlo realizations.

We concentrate on a scenario where $L=2$ delayed arrivals of a known signal are received by a uniform linear array with 6 antennas spaced $0.5 \lambda$ apart. This known signal is a concatenation of $K$ truncated and sampled Nyquist square root raised cosine pulses. Each pulse has a bandwidth equal to $(1+\alpha) / 2 T_{c}$, is truncated to the interval $\left[-5 T_{c}, 5 T_{c}\right]$, and the sampling period is $T_{s}=T_{c} / 2$, so there are 21 samples in each pulse. The roll-off factor is set equal to $\alpha=0.2$. The use of this type of signal is of interest because each pulse may represent the output of the despreader at every symbol period in a direct-sequence CDMA system. For simplicity, the spatial signatures of the two arrivals are the array steering vectors for DOAs equal to $0^{\circ}$ and $10^{\circ}$ relative to the broadside. The noise plus interference field in which the array operates consists of: i) spatially and temporally white Gaussian noise, and ii) a temporally white Gaussian interference at DOA $-30^{\circ}$. The remaining scenario parameters, except when one of them is varied, are as follows: $K=4$ pulses; delays of the two arrivals equal to $\tau_{1}=0$ and $\tau_{2}=0.5 T_{c}$; signal to noise ratio (SNR) of the first arrival: 14dB; Signal to Interference Ratio (SIR) of the first arrival: $-7 \mathrm{~dB}$; the second signal is attenuated $3 \mathrm{~dB}$ with respect the first, and they are in phase at the first sensor. The temporal spacing of the FIR channel is assumed to be $T_{0}=0.5 T_{c}$.

In figure 1 , the finite-sample and asymptotic performance in absence of model errors (i.e., $T_{0}=\tau_{1}-\tau_{0}$ and the length of the FIR filter $d$ is equal to the number of arrivals) of the different estimator is illustrated. We consider that the number of taps of the channel is $d=2$. The RMSEs of the exact ML estimator and the proposed approximation reach the CRB for small sample sizes. This fact proves that neither the approximation leading to (19) nor the subsequent minimization using the polynomial rooting algorithm entail a significant degradation with respect to the exact search-based estimator. Figure 2 bears out that the methods that take into account the spatial correlation of the interference are practically insensitive to the CCI level, whenever enough degrees of freedom are available. On the other hand, under the rather usual of assumption of white-noise, the resulting estimator completely fails for $\mathrm{SIR}<-10 \mathrm{~dB}$. In figure 3 , we investigate the performance of the estimators when for $d=4$ and the delay difference between the signal arrivals, $\tau_{2}-\tau_{1}$, does not necessarily coincide with the spacing of the FIR channel, $T_{0}$. As expected, the RMSE presents minima when the former is a multiple of the latter. In the other cases, the model in (1) is only approximate, which results in a higher RMSE. Finally, increasing the length of the FIR filter beyond the necessary minimum ( $d=2$ in this case) impairs the performance, as shown in figure 4 .

\section{CONCLUSIONS}

The problem of time delay estimation in a multipath channel has been considered. The channel is modeled as an unknown FIR filter, and the CCI is assumed to have unknown spatial correlation. Starting from the exact ML solution, we have derived an approximate estimator, which has allowed us to use a polynomial rooting approach to obtain the estimates. The proposed method attains the CRB in absence of modeling errors and is robust against arbitrarily high interference levels. Finally, the effects of varying the number of taps of the channel and varying the delay between the arrivals of the signal have been investigated.

\section{REFERENCES}

[1] S. Parkvall, E. Ström, and B. Ottersten, "The Impact of Timing Errors on the Performance of Linear DSCDMA Receivers," IEEE Journal on Selected Areas on Communications, vol. 14, pp. 1660-1668, Oct. 1996. 


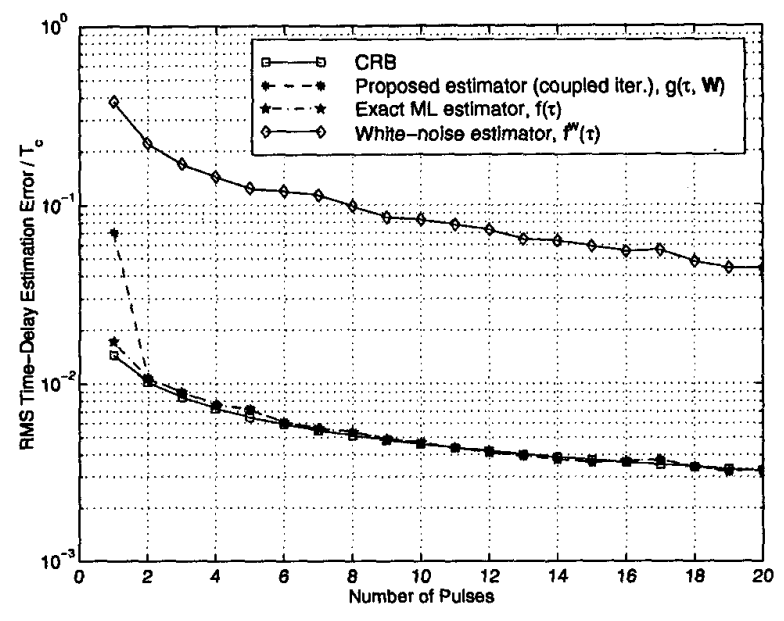

Figure 1: RMSE versus the number of training pulses.

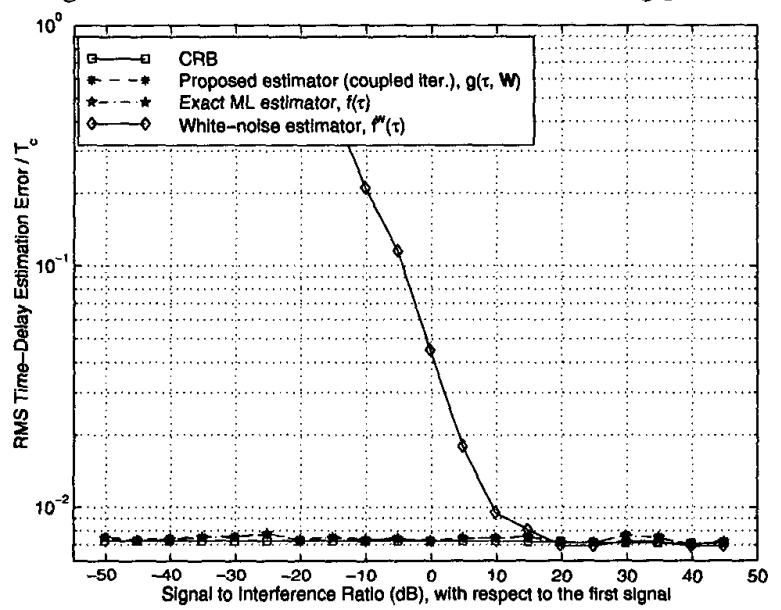

Figure 2: RMSE versus the interference power.

[2] M. Mengali and A. N. D'Andrea, Synchronization Techniques for Digital Receivers. Plenum Press, 1997.

[3] D. Astély, A. Jakobsson, and A. Swindlehurst, "Burst Synchronization on Unknown Frequency Selective Channels with $\mathrm{Co}$-Channel Interference Using an Antenna Array," in Proc. IEEE Vehicular Technology Conf., (Houston, TX), March 1999.

[4] G. Seco, A. Swindlehurst, J. A. Fernández-Rubio, and D. Astély, "A Reduced-Complexity and Asymptotically Efficient Time-Delay Estimator," in Proc. ICASSP, (Istambul, Turkey), 2000.

[5] G. Seco, A. L. Swindlehurst, and D. Astély, "Exploting Antenna Arrays for Synchronization," in Signal Processing Advances in Wireless Communications (G. B. Giannakis, Y. Hua, P. Stoica, and L. Tong, eds.), vol. II: Trends in Single- and Multi-User Systems, ch. 10, Prentice-Hall, 2000. To be published.

[6] A. L. Swindlehurst, "Time Delay and Spatial Signa-

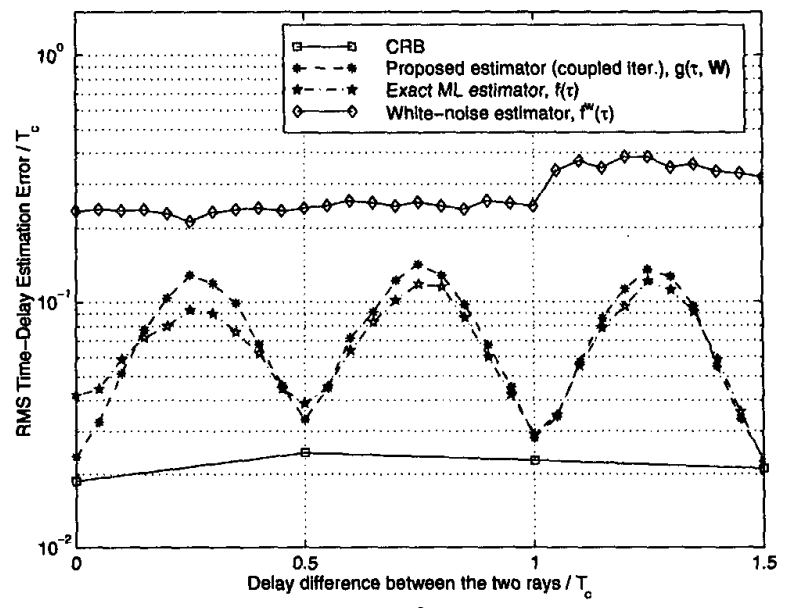

Figure 3: RMSE versus delay separation of the two arrivals.

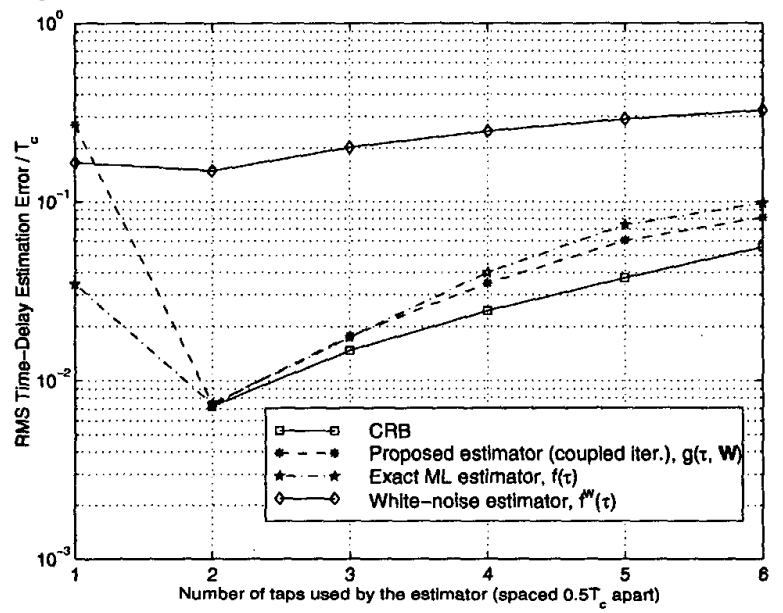

Figure 4: RMSE versus number of taps of the channel (d).

ture Estimation Using Known Asynchronous Signals," IEEE Trans. SP, vol. 46, pp. 449-462, Feb. 1998.

[7] M. Wax and A. Leshem, "Joint Estimation of Time Delays and Directions of Arrival of Multiple Reflections of a Known Signal," IEEE Trans. on SP, vol. 45, pp. 2477-2484, Oct. 1997.

[8] G. Raleigh and T. Boros, "Joint Space-Time Parameter Estimation for Wireless Communication Channels," IEEE Trans. on SP, vol. 46, pp. 1333-1343, May 1998.

[9] B. Fleury, M. Tshudin, R. Heddergott, D. Dahlhaus, and $\mathrm{K}$. Pedersen, "Channel Parameter Estimation in Mobile Radio Environments Using the SAGE Algorithm," IEEE J. Select. Areas Commun., vol. 17, pp. 434-450, March 1999.

[10] T. I. Laakso, V. Välimäki, M. Karjalainen, and U. K. Laine, "Splitting the Unit Delay," IEEE Signal Processing Magazine, vol. 13, pp. 30-60, Jan. 1996. 